Research Paper

\title{
Carbon Nanotube Thin Film Biosensors for Sensitive and Reproducible Whole Virus Detection
}

\author{
Himadri S. Mandal, Zhengding Su, Andrew Ward, Xiaowu (Shirley) Tang ${ }^{凶}$ \\ Department of Chemistry, University of Waterloo, 200 University Avenue West, Waterloo, Ontario, N2L 3G1, Canada.
}

$\triangle$ Corresponding author: X. S. Tang, Department of Chemistry, University of Waterloo, 200 University Ave West, Waterloo, ON, Canada N2L 3G1 Email: tangxw@uwaterloo.ca Phone: 519-888-4567 ext. 38037 Fax: 519-746-0435

(C) Ivyspring International Publisher. This is an open-access article distributed under the terms of the Creative Commons License (http://creativecommons.org/ licenses/by-nc-nd/3.0/). Reproduction is permitted for personal, noncommercial use, provided that the article is in whole, unmodified, and properly cited.

Received: 2011.10.28; Accepted: 2011.12.02; Published: 2012.03.01

\begin{abstract}
Here, we report the label-free, sensitive, and real-time electrical detection of whole viruses using carbon nanotube thin film (CNT-TF) field effect devices. Selective detection of approximately 550 model viruses, M/3-bacteriophage, is demonstrated using a simple two-terminal (no gate electrode) configuration. Chemical gating through specific antibody-virus binding on CNT surface is proposed to be the sensing mechanism. Compared to electrical impedance sensors with identical microelectrode dimensions (no CNT), the CNT-TF sensors exhibit sensitivity 5 orders higher. We believe the reported approach could lead to a reproducible and cost-effective solution for rapid viral identification.
\end{abstract}

Key words: carbon nanotube, thin film, virus detection, electrochemical impedance sensor, microelectrodes, M13-pIII antibody, M13 bacteriophage, ELISA.

\section{Introduction}

Rapid and accurate identification of viral pathogens is of vital importance to both medical diagnosis and biodefense. A variety of novel sensing techniques have been developed for this purpose during the past decade, which have demonstrated remarkably high sensitivity and great potential for miniaturization. However, the majority of sensing techniques identify and characterize viruses through detection of their signature proteins [1], [2], [3], [4], [5], [6], [7] or DNA [6], [8], [9], [10], [11], [12], which still requires complex sample preparation steps including viral isolation, extraction, purification, and amplification of respective biomolecules. In contrast, whole-virus sensors require much simpler sample preparation and are therefore potentially more suited for integration into lab-on-a-chip devices for point-of-care or in-field analysis. Whole-virus sensing based on specific antibody recognition is especially promising, due to the rapid recent advancements in design and production of recombinant antibodies [13], [14], [15].

To date, a few platforms, including whispering-gallery microlasers [16], opto-fluidic micro-ring resonators [17], silicon nanowire FETs [18], and surface plasmon resonance [19], have been reported with the ability to detect a single influenza A virus in a buffered or serum solution, which outlines the immense potential of whole-virus detection techniques. Compared to standard viral detection methods based on genetic analysis and immunoassays, however, the above-mentioned platforms generally suffer from poor reproducibility and high cost [20], [21], [22], [23].

Others have reported whole-virus detection of T7 bacteriophage using CNT-TF [24] as well as polymer nanowire [25] platforms. The solution based CNT-TF deposition methods described in [24] are known to yield inferior films in terms of mechanical 
strength and are not compatible with the lithographic or transfer [26] protocol. Further, this CNT-TF synthesis involved complicated circuitry for AC dielectrophoresis as well as dissolved and sonicated (damaged) CNTs. The polymer nanowire platform described in [25] utilized nanowires with a large variation in size (and in turn the conductivity and reproducibility) and had issues such as swelling (due to inclusion of solvents and electrolytes) and large hysteresis.

In our approach, CNT-TF synthesis involves the most economically viable carbon source (alcohol vapor) and bypasses the laborious and time-consuming purification required to prepare solution based CNT films. We describe the fabrication of reproducible sensors (following the conventional semiconductor processing protocol) for label-free and real-time electrical detection of whole viruses in a biocompatible buffer solution. In this respect, our novel approach involving CNT synthesis and sensor design attempts to achieve the best combined performance in terms of sensitivity, reproducibility, and cost.

\section{Materials and Methods}

\section{Fabrication of CNT thin film Sensors}

Homogeneous and transferable CNT thin films (CNT-TFs) were synthesized using a previously reported chemical vapor deposition (CVD) method [27]. The CNT-TFs were then transferred, with a poly(methyl methacrylate) (PMMA) thin film support, from growth substrates to $\mathrm{SiO}_{2}(500 \mathrm{~nm}) / \mathrm{p}^{++}-\mathrm{Si}$ substrates pre-patterned with source and drain electrodes $(50 \mathrm{~nm} \mathrm{Au}$ on $2 \mathrm{~nm} \mathrm{Cr}$ ). Using a negative photoresist (AZnLOF, MicroChemicals $\mathrm{GmbH}$ ) and photolithography, blocks of CNT-TFs bridging the electrodes were protected and the exposed film was etched away by reactive ion etching (RIE). A rather harsh condition (heating in DMSO at $180^{\circ} \mathrm{C}$ for $8 \mathrm{hrs}$ ) was then used to strip PMMA and photoresist off the CNT-TF blocks to ensure clean CNT surface.

\section{Phage Preparation}

M13 phage, as a model target virus, was prepared by transfecting M13KE phagemid (New England Lab, MA) into E. coli 2738 competent cells (New England Lab, MA). The transfected E. coli strains were spread on an LB/IPTG/Xgal plate. The plate was incubated at $37^{\circ} \mathrm{C}$ overnight to generate phage plaques. A single plaque was picked up and inoculated into 1 $\mathrm{mL}$ of E. coli cell culture, which was diluted from overnight culture with LB medium in a ratio of 1:100. The inoculated cell culture was incubated at $37^{\circ} \mathrm{C}$ with shaking for 5 hours, followed by centrifugation. The supernatant was subjected to precipitation with
$20 \%$ PEG / $2.5 \mathrm{M} \mathrm{NaCl}$ for $20 \mathrm{~min}$ at room temperature. Precipitated phages were collected by centrifugation and phage concentration was determined by phage titration. Phage pellets were dissolved in 10 $\mathrm{mM}$ phosphate buffer ( $\mathrm{pH} 7.5$ ) containing $150 \mathrm{mM}$ $\mathrm{NaCl}$ and $5 \%$ glycerol. An aliquot of phage solution was used for further amplification of phage in order to gain a high concentration stock of M13 phage ( $10^{10} \mathrm{pfu} / \mathrm{mL}$ ).

\section{Confirmation of phage binding on CNT thin film}

Phage-bound CNT thin films were scratched from $\mathrm{SiO}_{2}$ substrates and transferred to an eppendorf tube, which were then blocked with $1 \%$ BSA in a buffer containing $150 \mathrm{mM} \mathrm{NaCl}, 5 \%$ glycerol at $4{ }^{\circ} \mathrm{C}$ overnight. Bound phages were then eluted by incubating the films in $100 \mu \mathrm{L}$ of $1 \mathrm{mg} / \mathrm{mL}$ BSA in $2 \mathrm{M}$ glycine- $\mathrm{HCl}(\mathrm{pH} 2.2)$ for $5 \mathrm{~min}$. The eluted phages were immediately neutralized by adding $15 \mu \mathrm{L}$ of $1 \mathrm{M}$ Tris- $\mathrm{HCl}(\mathrm{pH} 9.1)$ and the phage concentration was scored by titration using E. coli strain ER2738.

\section{Results and Discussion CNT Thin Film (CNT-TF) Sensors}

Silicon oxide chips $\left(1 \mathrm{~cm}^{2}\right)$, each with an array of 120 CNT-TF devices, were fabricated as described in the Materials and Methods section. Each device consists of a rectangular block of semiconducting CNT-TF bridging a pair of Au source/drain electrodes $(100 \mu \mathrm{m}$ wide). The devices are in a microfluidic compatible layout with $\mathrm{CNT}$ conductance channel lengths (i.e. gap between each pair of source/drain electrodes) varying from $3 \mu \mathrm{m}$ to $100 \mu \mathrm{m}$ (Figure 1a). Using the highly doped $\mathrm{Si}$ underneath $\mathrm{SiO}_{2}$ as a back gate, the electrical properties of the sensors were tested in a dry state. The devices with $100 \mu \mathrm{m}$ channel length showed the best reproducibility from batch to batch, which were within $10 \%$ to $15 \%$ in terms of switching (on/off ratio $\sim 2.3)$ and on-state conductance $(\sim 55 \mathrm{k} \Omega)$ respectively (Figure $1 \mathrm{~b}$ ), and therefore were used exclusively for whole virus sensing in this study.

To demonstrate the direct electrical detection of viruses in biological compatible buffer solution, a polydimehtylsiloxane (PDMS) slab was bonded onto the chip to form a microfluidic channel across the array of CNT-TF sensors. While analyte solution was delivered through the microfluidic channel, a constant source-drain voltage $\left(\mathrm{V}_{\mathrm{sd}}=10 \mathrm{mV}\right)$ was simultaneously applied to the sensors and the source-drain current $\left(\mathrm{I}_{\mathrm{sd}}\right)$ was recorded as a function of time $(\mathrm{I}-\mathrm{t})$. There was no solution gate involved, therefore our CNT-TF devices function as simple two-terminal electrical impedance sensors (EIS). 

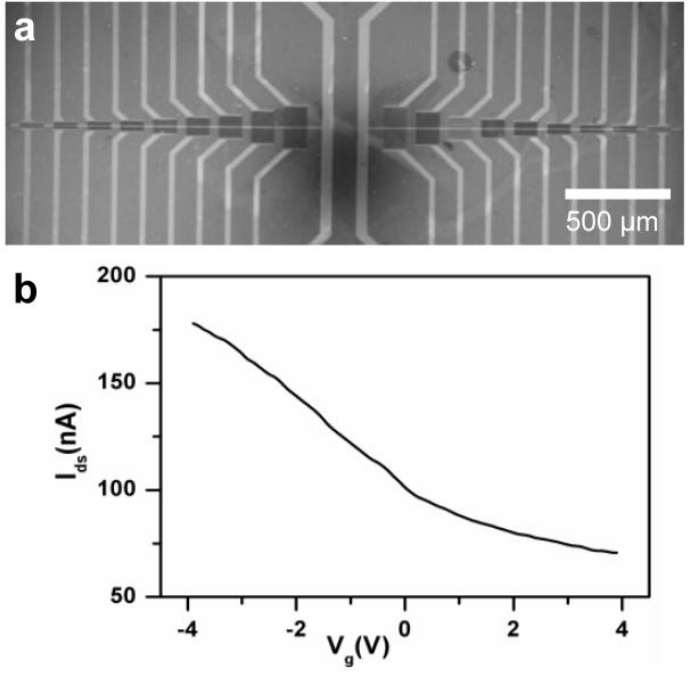

Figure I. (a) SEM image of a CNT thin film sensor array. (b) I-Vg curve showing typical switching property of a sensor with $100 \mu \mathrm{m}$ channel length $\left(\mathrm{V}_{\mathrm{sd}}=10 \mathrm{mV}\right)$.

A non-lytic bacterial virus, M13 bacteriophage, which uses its minor coat protein pIII to attach to the receptors at the tip of the $\mathrm{F}$ pilus region of the host Escherichia coli for transfection, was used as a model target virus. To selectively detect M13, a CNT-TF sensor was first equilibrated in 1x PBS buffer followed by the addition of M13-pIII antibodies (which specifically bind to the pIII coat protein of M13) to yield a final concentration of $1 \mathrm{pM}$. To avoid nonspecific adsorption on the sensor surface, a solution of BSA (1\% in $1 x$ PBS) was flushed subsequently. After a wash with 1x PBS, M13 phage (ca. $0.5 \mathrm{pM}$ ) was introduced. The source-drain current $\left(\mathrm{I}_{\mathrm{sd}}\right)$ decreased slightly $(\sim$ $5 \%)$ in response to M13-pIII Ab and BSA, and then significantly $(>20 \%)$ in response to the target virus, M13-phage (Figure 2a). $\mathrm{I}_{\mathrm{sd}}$ did not reach a steady state during the 2 hours of monitored period. We suspect that the initial sharp decrease is reaction limited, which is due to capturing of phages very close to the sensor surface, and the later slow drift is due to diffusion-limited transport of phages [28]. To demonstrate the specificity of the CNT-TF sensor, two control experiments were carried out. M13-pIII Ab modified CNT-TF sensor showed negligible response to an anti-M13 phage (as a control virus model), which does not have pIII coat protein. Blocking of M13-pIII antibodies on the sensor surface by a secondary anti-mouse IgG prior to the introduction of M13 phage also rendered the sensor irresponsive. Figure $2 b$ shows the overall electrical response of CNT-TF sensors in the three scenarios, for which the binding of antibodies and viruses on a sensor surface are illustrated schematically in Figure 2c. It is worth noting is that the PDMS microfluidic channel width is less than $100 \mu \mathrm{m}$, therefore only the CNT network (not the gold electrodes) were exposed in solution. CNT served as the anchor for M13-pIII Ab immobilization and the transducer, which translated the specific binding between M13-pIII Ab and M13 phage to a change in electrical signal in a user-friendly two-terminal configuration.

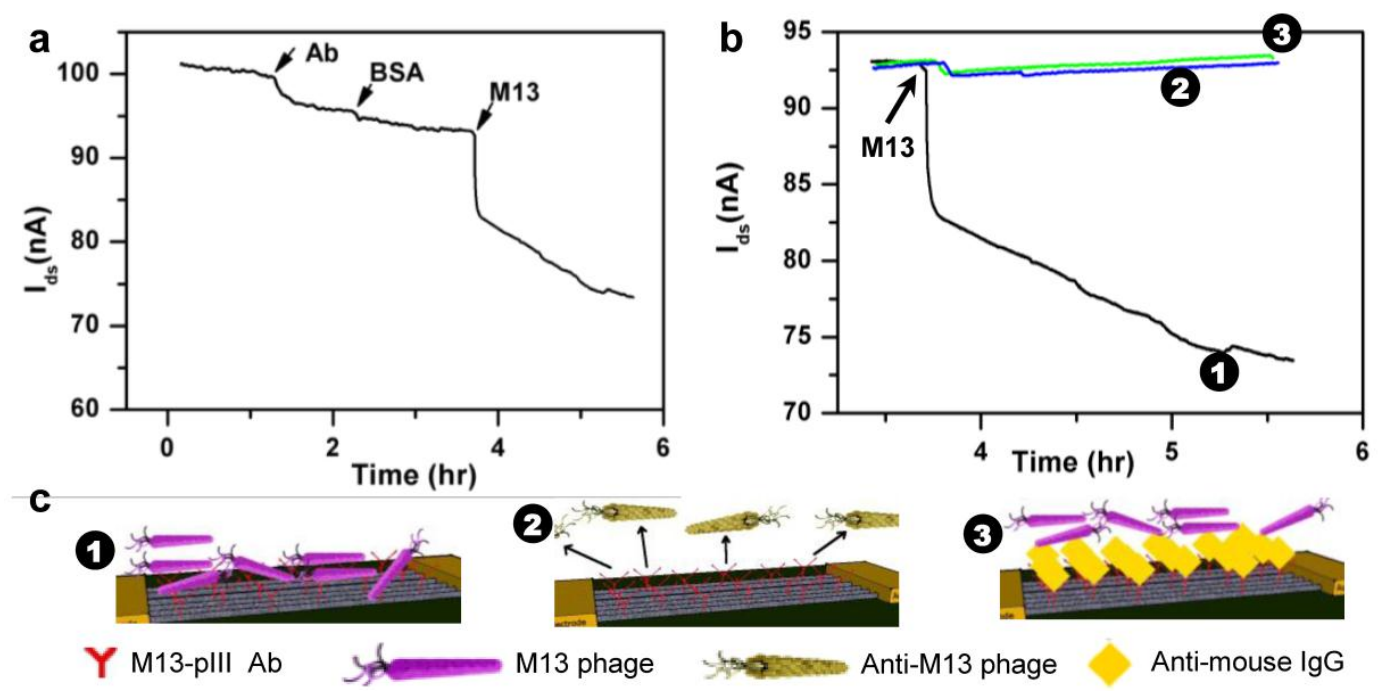

Figure 2. (a) I-t measurement of the response of a CNT-TF sensor to MI3-plll antibody, BSA, and MI 3 phage in I $\times$ PBS. (b) Response of a MI3-plll Ab and BSA coated CNT-TF sensor to MI3 phage (I) in comparison with two controls: with exposure to anti-MI3 phage (2) and with MI3-plll Ab blocked prior to exposure to MI3 phage (3). (c) Schematic illustration of the corresponding scenarios. 



Figure 3. AFM images of a CNT network on a $\mathrm{SiO}_{2}$ substrate after (a) MI3-plll modification, (b) BSA blocking, and (c) capturing MI3 phages.

Atomic force microscopy (AFM) was then used to verify the adsorption of proteins and capture of viruses at various steps on a $\mathrm{CNT}-\mathrm{TF} / \mathrm{SiO}_{2}$ substrate (Figure 3). Figure 3a shows the decoration of M13-pIII $\mathrm{Ab}$ (small white spots) on the sidewall of long (a few $\mu \mathrm{m})$ and interconnected CNTs, as well as on $\mathrm{SiO}_{2}$, after emerging the substrate into an M13-pIII Ab solution. The resulting tube density is approximately 10 tubes $/ \mu \mathrm{m}^{2}$. After BSA passivation (Figure $3 b$ ), string-like M13 were shown to be captured on the surface of either CNTs or $\mathrm{SiO}_{2}$ (Figure 3c), which confirmed the retention of phage-binding activity of the surface adsorbed M13-pIII antibodies. An M13 phage typically has a diameter of $5 \mathrm{~nm}$ and a length around $800 \mathrm{~nm}$ [29], [30]. The activity of CNT-bound M13-pIII antibodies, in particular, were further confirmed by enzyme-linked immunosorbent assay (ELISA) (see ESM). The exposure conditions of the $\mathrm{CNT} / \mathrm{SiO}_{2}$ substrate to M13-pIII Ab, BSA, and M13 phage are identical to those used for electrical detection described in the previous paragraph.

By phage titration, the number of M13 captured on a piece of M13-pIII Ab coated CNT thin film $(\sim 5$ $\mathrm{mm}^{2}$ ) was determined to be about 550 after 500 times dilution (Figure 4). Therefore, the original density of CNT-bound viruses was 55,000 per $\mathrm{mm}^{2}$. Since each sensor consists of a $100 \mu \mathrm{m} \times 100 \mu \mathrm{m}$ CNT-TF, we conclude that our sensors are well capable of label-free and real-time detection of approximately 550 viruses in a biocompatible buffer. The control CNT thin film, which is BSA blocked and without M13-pIII $\mathrm{Ab}$, is virus free. The error bar indicates the standard deviation of phage plaque count (pfu) titrated from phages eluted from five CNT thin films with the same area and tube density.

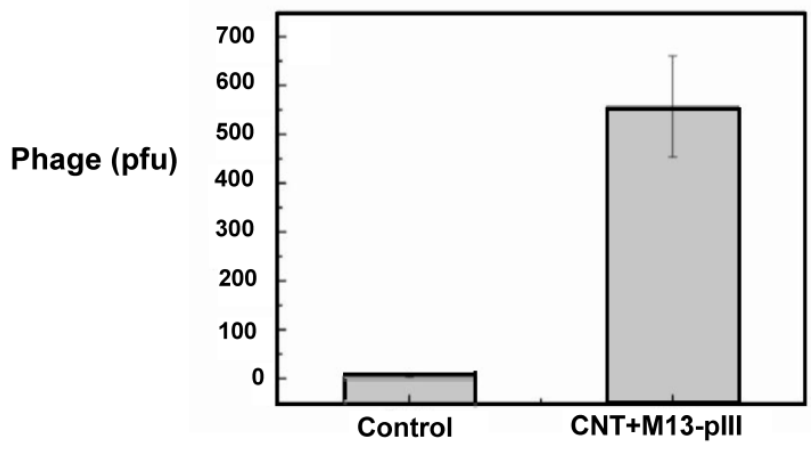

Figure 4. Phage plaque count titrated from phages captured on an MI3-plll Ab coated $5 \mathrm{~mm}^{2}$ CNT-TF.

\section{CNT-TF Sensor Design Rationale}

A CNT-FET containing a single semiconducting single-walled CNT is considered the ideal sensor that offers ultimate miniaturization and sensitivity. However, CNT sensors with a single or a few nanotubes have two main drawbacks. First, mass-producing such devices in a reproducible way is impossible to date due to the variation in nanotube chirality, diameter, and growth yield [31]. Second, typical single- or few-tube devices have CNTs interconnect a pair of closely spaced (less than $2 \mu \mathrm{m}$ apart) source/drain micro-electrodes that are also exposed in the analyte solution, which could compromise the sensor sensitivity and specificity. The size of the microelectrodes and the gap between them are commonly limited by the length of individual CNTs and the complexity of micro/nano-fabrication processes.

It is well known that biomolecules and pathogenic particles interact not only with CNTs, but also with metal electrode surfaces [32]. Figure 5a shows the equivalent circuit of a CNT-FET sensor with electrodes in solution, where $\mathrm{C}_{\mathrm{dl}}$ is the double layer ca- 
pacitor formed in the vicinity of the metal electrodes, $R_{\text {sol }}$ and $R_{C N T}$ are the resistance of the solution and CNT conduction channels respectively. With electrodes immersed in solution, the overall sensor can be modeled as a simple microelectrode EIS (ME-EIS) in parallel with a CNT-FET. To demonstrate experimentally, we fabricated pairs of Au electrodes (100 $\mu \mathrm{m}$ wide and $100 \mu \mathrm{m}$ apart) without CNTs, i.e. ME-EISs, and performed electrical sensing (Figure 5). While maintaining $\mathrm{V}_{\mathrm{sd}}=10 \mathrm{mV}, \mathrm{I}_{\mathrm{sd}}$ spiked up upon immersion into 1x PBS, which is a clear evidence of $\mathrm{C}_{\mathrm{dl}}$ formation (Figure $5 \mathrm{~b}$ ). The current gradually decreases to a steady-state value set by the resistance $\mathrm{R}_{\text {sol, }}$ which is inversely proportional to electrode area (Figure 5c) and ionic strength, and proportional to the distance between the pair of electrodes. Apparently, adsorption and binding of analytes on the microelectrode surface will introduce (1) a transient response in $\mathrm{I}_{\mathrm{sd}}$ due to the change to the double layer capacitance $\mathrm{C}_{\mathrm{dl}}$, and (2) a steady-state response due to the change to $R_{\text {sol. }}$. As shown in Figure $5 d$, the ME-EIS responses effectively to $100 \mathrm{nM}$ M13-pIII Ab and $50 \mathrm{nM}$ M13 phage, which demonstrated sensitivity consistent with traditional EIS with similar microelectrode geometries. If a CNT-FET sensor had $R_{\text {CNT }}$ compatible to or even higher than $R_{\text {sol }}(10-100 \mathrm{k} \Omega$ in a biocompatible buffer), as in the case of single- or a few-nanotube devices, sensitivity and specificity will be limited by
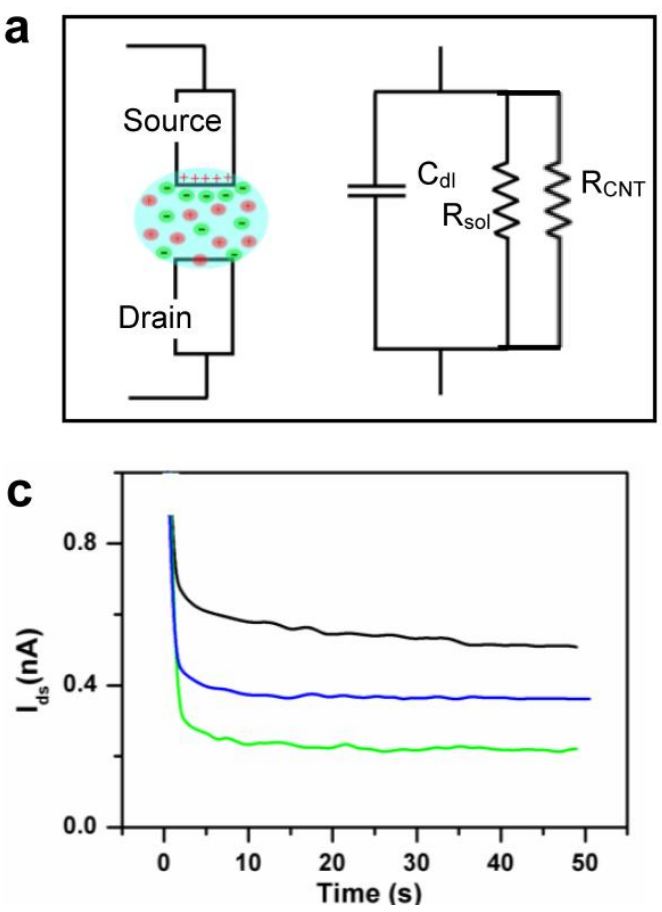

adsorption and binding of analytes on electrodes. In order to take use of the unique size, surface, and electrical properties of CNTs for high sensitivity detection, a CNT sensor must have $\mathrm{R}_{\mathrm{CNT}}<<\mathrm{R}_{\text {sol }}$, or alternatively avoid exposing the electrodes in analyte solutions.

By using CNT thin films, instead of single or a few CNTs, one can effectively address the aforementioned drawbacks faced by single or few CNT-FET sensors. Besides high sensitivity, a few factors were taken into account in the CNT-TF sensor design in this study, which are low-cost, reproducibility, and easy integration with microfluidic channels. CNT thin films were synthesized using alcohol vapor, a relatively low-cost and high yield carbon source. Time-consuming and expensive purification protocols were avoided. The tube density $\left(\sim 10\right.$ tubes $\left./ \mu \mathrm{m}^{2}\right)$ and sensor dimension $(100 \mu \mathrm{m} \times 100 \mu \mathrm{m})$ were carefully balanced for optimized reproducibility and sensing performance. The relatively large sensor dimension simplifies fabrication and makes it easy to couple the sensors with microfluidic channels while exposing only the CNTs, not the metal electrodes in analyte solutions. With identical electrical sensing configurations, the sensitivity of a CNT-TF sensor (Figure 2a), which is down to 1 pM M13-pIII Ab and 0.5 pM M13, is 5 orders higher than that of a ME-EIS of the same dimensions (Figure 5d).



Figure 5. (a) A schematic representation and the equivalent circuit of a CNT sensor. (b) Initial spike and (c) the steady state current across a pair of source/drain electrodes (without CNT) upon merging of a dry device into I $\times$ PBS. The steady state current is a function of electrode area exposed in solution: ca. $1000 \mu \mathrm{m}^{2}$ (green), ca. $2000 \mu \mathrm{m}^{2}$ (blue), and ca. $3000 \mu \mathrm{m}^{2}$ (black). (d) Electrical response of a device without CNTs (electrodes only) to the addition of MI3-plll antibody, BSA, and MI 3 phage in I $\times$ PBS. 


\section{Sensing Mechanism}

Three sensing mechanisms were proposed for CNT field effect sensors in various literature reports, which are capacitive gating, doping / chemical gating, and Schottky barrier (SB) modulation. In this study, we believe chemical gating [33], [34], [35] is the dominant mechanism.

In previous studies, either the highly doped $\mathrm{Si}$ was used as a back gate, or an $\mathrm{Ag} / \mathrm{AgCl}$ reference [36], [37] or Pt pseudo-reference electrode [38], [39], [40] was used as a solution gate during electrical sensing. In this study, gate electrodes were eliminated through the use of microfluidic channels. Not only do we provide a simpler configuration, but the effect of capacitive gating suggested in the study by Besteman et al. [36], can also be ruled out. The source-drain conductance of a CNT-FET is known to be insensitive to back gate once immersed in aqueous solution and biased by adsorption on reference electrodes. SB modulation at the metal-CNT contact due to the change of metal work function, as suggested in the studies by Chen et al. [38], [40], can also be ruled out since only CNTs were exposed to analytes in this study. However, modulation of the barrier height at CNT-CNT intersections could potentially contribute to the overall conductance change of our CNT thin films, which awaits further investigation.

Chemical gating or doping originates from direct binding or close contact (within nanometers) of analyte molecule/particles to CNTs, which often introduces localized scattering centers, as well as depletion or enrichment of charge carriers in CNTs. Most commonly, chemical gating leads to a decrease in CNT conductance due to increased scattering, as observed in this study. The response of our CNT-TF sensors to M13-pIII Ab and BSA can be explained by the direct binding of protein molecules on the CNT sidewall. The more significant response to M13 phage can be attributed to the conformational change of CNT-bound M13-PIII Ab caused by the specific phage- $\mathrm{Ab}$ binding [41], [42] and possibly multiple binding sites per phage.

\section{Conclusions}

In this work, we demonstrated real-time label-free detection of whole viruses in a biocompatible buffer solution using an antibody-based CNT-TF sensor. Compared to previously reported CNT-FET sensors with single or a few nanotubes, CNT-TF sensors are more reproducible and easier to both fabricate and integrate with microfluidic channels. We showed experimentally that metal electrodes exposed in the analyte solution not only have a role in detec- tion, but also hinder sensitivity and selectivity of a CNT-FET sensor. Compared to ME-EIS of the same dimension, our CNT-TF sensors require much less reagent (i.e. surface anchored antibodies) and have a sensitivity 5 order of magnitude higher. Electrical response to about 550 viruses on a sensor surface was recorded. We propose "chemical gating" as the dominant sensing mechanism, which originates from direct contact/binding of analytes to CNTs. Therefore, high sensitivity can be achieved in buffers with high ionic strength, which typically hinders capacitive gating [36], [43], [44]. Also, in our configuration, the CNT-TF sensors are two-terminal (no gate). So low-cost substrates (e.g. glass, paper) can be used as the alternative to the expensive Si. Combining with microfluidics, advancements in recombinant antibody design and production, our CNT-TF whole-virus sensors could offer a cost-effective solution for rapid viral identification based on specific antibody recognition.

\section{Supplementary Material}

Electronic Supplementary Material. http://www.thno.org/v02p025 I I I.pdf

\section{Acknowledgements}

This project was supported by a Discovery grant and a Strategic grant from the Natural Sciences and Engineering Research Council (NSERC) of Canada.

\section{Conflict of Interest}

The authors have declared that no conflict of interest exists.

\section{References}

1. Abe M, Murata K, Kojima A, Ifuku Y, Shimizu M, et al. Quantitative detection of protein using a top-gate carbon nanotube field effect transistor. J Phys Chem. 2007; 111: 8667-8670.

2. Long $Y$, Zhang Z, Yan X, Xing J, Zhang K, et al. Multiplex immunodetection of tumor markers with a suspension array built upon core-shell structured functional fluorescence-encoded microspheres. Anal Chim Acta. 2010; 665: 63-68.

3. Su L, Chen R, Li Y, Chang Y, Lee Y, et al. Detection of prostate-specific antigen with a paired surface plasma wave biosensor. Anal Chem. 2010; 82: 3714-3718.

4. Zhang B, Zhang X, Yan H, Xu S, Tang D, et al. A novel multi-array immunoassay device for tumor markers based on insert-plug model of piezoelectric immunosensor. Biosens Bioelectron. 2007; 23: 19-25.

5. Palaniappan A, Goh WH, Tey JN, Wijaya IPM, Moochhala SM, et al. Aligned carbon nanotubes on quartz substrate for liquid gated biosensing RID A-2231-2011. Biosens Bioelectron. 2010; 25: 1989-1993.

6. Lechuga LM, Tamayo J, Alvarez M, Carrascosa LG, Yufera A, et al. A highly sensitive microsystem based on nanomechanical biosensors for genomics applications. Sens Actuat B: Chem. 2006; 118: 2-10. 
7. Lee JH, Hwang KS, Park J, Yoon KH, Yoon DS, et al. Immunoassay of prostate-specific antigen (PSA) using resonant frequency shift of piezoelectric nanomechanical microcantilever. Biosens Bioelectron. 2005; 20: 2157-2162.

8. Star A, Tu E, Niemann J, Gabriel JCP, Joiner CS, et al. Label-free detection of DNA hybridization using carbon nanotube network field-effect transistors. Proc Natl Acad Sci USA. 2006; 103: 921-926.

9. Giraud G, Schulze H, Bachmann TT, Campbell CJ, Mount AR, et al. Solution state hybridization detection using time-resolved fluorescence anisotropy of quantum dot-DNA bioconjugates. Chem Phys Lett. 2010; 484: 309-314.

10. Chiu C, Lee H, Kuo C, Gwo S. Immobilization of DNA-au nanoparticles on aminosilane-functionalized aluminum nitride epitaxial films for surface acoustic wave sensing. Appl Phys Lett. 2008; 93: 1-3.

11. Baur J, Gondran C, Holzinger M, Defrancq E, Perrot H, et al. Label-free femtomolar detection of target DNA by impedimetric DNA sensor based on poly(pyrrole-nitrilotriacetic acid) film. Anal Chem. 2010; 82: 1066-1072.

12. Zhang J, Lang HP, Huber F, Bietsch A, Grange W, et al. Rapid and label-free nanomechanical detection of biomarker transcripts in human RNA. Nat Nanotech. 2006; 1: 214-220.

13. Peters J, Stoger E. Transgenic crops for the production of recombinant vaccines and anti-microbial antibodies. Hum Vaccines. 2011; 7: 367-374.

14. Yusibov V, Streatfield SJ, Kushnir N. Clinical development of plant-produced recombinant pharmaceuticals vaccines, antibodies and beyond. Hum Vaccines. 2011; 7: 313-321.

15. Schirrmann T, Al-Halabi L, Duebel S, Hust M. Production systems for recombinant antibodies. Front Biosci. 2008; 13: 4576-4594.

16. Vollmer F, Arnold S, Keng D. Single virus detection from the reactive shift of a whispering-gallery mode. Proc Natl Acad Sci USA. 2008; 105: 20701-20704.

17. Zhu H, White IM, Suter JD, Zourob M, Fan X. Opto-fluidic micro-ring resonator for sensitive label-free viral detection. Analyst. 2008; 133: 356-360.

18. Patolsky F, Zheng GF, Hayden O, Lakadamyali M, Zhuang XW, et al. Electrical detection of single viruses. Proc Natl Acad Sci USA. 2004; 101: 14017-14022.

19. Wang S, Shan X, Patel U, Huang X, Lu J, et al. Label-free imaging, detection, and mass measurement of single viruses by surface plasmon resonance. Proc Natl Acad Sci USA. 2010; 107: 16028-16032.

20. Caygill RL, Blair GE, Millner PA. A review on viral biosensors to detect human pathogens. Anal Chim Acta. 2010; 681: 8-15.

21. Bellan LM, Wu D, Langer RS. Current trends in nanobiosensor technology. Wil Interdis Rev: Nanomed and Nanobio. 2011; 3: 229-246.

22. Justino CIL, Rocha-Santos TA, Duarte AC. Review of analytical figures of merit of sensors and biosensors in clinical applications. Trends Anal Chem. 2010; 29: 1172-1183.

23. D'Orazio P. Biosensors in clinical chemistry-2011 update. Clin Chim Acta. 2011; 412: 1749-1761.

24. Garcia-Alijaro C, Cella LN, Dhammanand JS, Mulchandani A. Carbon nanotubes-based chemiresistive biosensors for detection of microorganisms. Biosens Bioelectron. 2010; 26: 1437-1441.

25. Dhammanand S, Bangar MA, Park M, Mulchandani A. Label-free chemiresistive immunosensors for viruses. Environ Sci Technol. 2010; 44: 9030-9035.

26. Sangwan VK, Southard A, Moore TI, Williams ED. Transfer printing approach to all-carbon nanoelectronics. Microelectron Eng. 2011; 88: 3150-3154.

27. Mandal HS, Ward A, Tang X. Transferable thin films of pristine carbon nanotubes. J Nanosci Nanotech. 2011; 11: 3265-3272.
28. Nair PR, Alam MA. Performance limits of nanobiosensors. App Phys Lett. 2006; 88:233120.

29. Beaudoin J, Henry TJ, Pratt D. Purification of single-length and double-length M13 virions by polyacrylamide-gel electrophoresis. J Virol. 1974; 13: 470-477.

30. Salivar W, Tzagoloff H, Pratt D. Some physical-chemical and biological properties of rod-shaped coliphage M13. Virol. 1964; 24: 359-371.

31. Kumar S, Cola BA, Jackson R, Graham S. A review of carbon nanotube ensembles as flexible electronics and advanced packaging materials. J Elec Pkg. 2011; 133: 1-12.

32. Davis JJ, Halliwell CM, Hill HAO, Canters GW, van Amsterdam MC, et al. Protein adsorption at a gold electrode studied by in situ scanning tunnelling microscopy. N J Chem. 1998; 22: 1119-1123.

33. Kong J, Franklin N, Zhou C, Chapline M, Peng S, et al. Nanotube molecular wires as chemical sensors. Science. 2000; 287: 622-625.

34. Star A, Han TR, Gabriel JCP, Bradley K, Gruner G. Interaction of aromatic compounds with carbon nanotubes: Correlation to the hammett parameter of the substituent and measured carbon nanotube FET response. Nano Lett. 2003; 3: 1421-1423.

35. Bradley K, Briman M, Star A, Gruner G. Charge transfer from adsorbed proteins. Nano Lett. 2004; 4: 253-256.

36. Besteman K, Lee JO, Wiertz FGM, Heering HA, Dekker C. Enzyme-coated carbon nanotubes as single-molecule biosensors. Nano Lett. 2003; 3: 727-730.

37. Heller I, Janssens AM, Mannik J, Minot ED, Lemay SG, et al. Identifying the mechanism of biosensing with carbon nanotube transistors. Nano Lett. 2008; 8: 591-595.

38. Chen R, Choi H, Bangsaruntip S, Yenilmez E, Tang X, et al. An investigation of the mechanisms of electronic sensing of protein adsorption on carbon nanotube devices. J Am Chem Soc. 2004; 126: 1563-1568.

39. Tang X, Bansaruntip S, Nakayama N, Yenilmez E, Chang Y, et al. Carbon nanotube DNA sensor and sensing mechanism. Nano Lett. 2006; 6: 1632-1636.

40. Kim S, Kim TG, Byon HR, Shin H, Ban C, et al. Recognition of single mismatched DNA using MutS-immobilized carbon nanotube field effect transistor devices RID C-6752-2011. J Phys Chem B. 2009; 113: 12164-12168.

41. Brun MA, Tan K, Nakata E, Hinner MJ, Johnsson K. Semisynthetic fluorescent sensor proteins based on self-labeling protein tags. J Am Chem Soc. 2009; 131: 5873-5884.

42. Wang W, Weng J, Zhang X, Liu M, Zhang M. Creating conformational entropy by increasing interdomain mobility in ligand binding regulation: A revisit to N-terminal tandem PDZ domains of PSD-95. J Am Chem Soc. 2009; 131: 787-796.

43. So H, Won K, Kim Y, Kim B, Ryu B, et al. Single-walled carbon nanotube biosensors using aptamers as molecular recognition elements. J Am Chem Soc. 2005; 127: 11906-11907.

44. Nair PR, Alam MA. Screening-limited response of nanobiosensors. Nano Lett. 2008; 8: 1281-1285. 